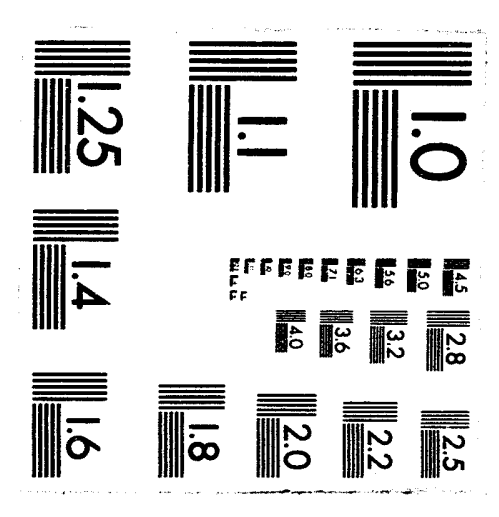



UCRL-JC-114300

PREPRINT

\title{
Applying Observations of Work Activity in Designing Prototype Data Analysis Tools
}

\author{
Rebecca R. Springmeyer
}

This paper was prepared for submittal to

Visualization' 93

San Jose, $C A$

October 25-29, 1993

July 6, 1993

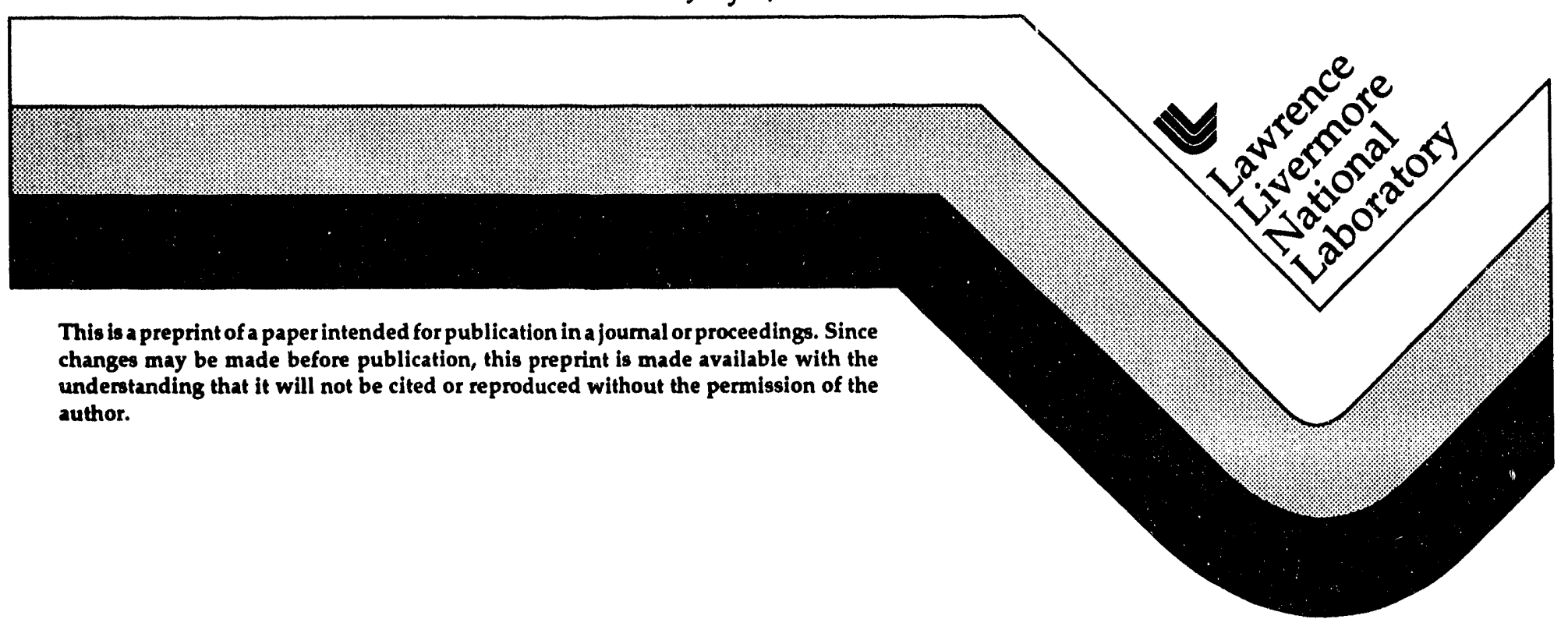




\section{DISCLAIMER}

This document was prepared as an account of work sponsored by an agency of the United States Government. Neither the United States Government nor the University of California nor any of their employees, makes any warranty, express or implied, or assumes any legal liability or responsibility for the accuracy, completeness, or usefulness of any information, apparatus, product, or process disclosed, or represents that its use woul d not infringe privately owned rights. Reference herein to any specific commercial paoducts, process, or service by trade name, trademark, manufacturer, or otherwise, does not necessarily constitute or imply its endorsement, recommendation, or favoring by the United States Government or the University of California. The views and opinions of authors expressed herein do not necessarily state or reflect those of the United States Government or the University of Califomia, and shall not be used for advertising or product endorsement purposes. 


\title{
Applying Observations of Work Activity in Designing rototype Data Analysis Tools
}

\author{
Rebecca R. Springmeyer \\ Lawrence Livermore National Laboratory \\ Livermore, California 94550 \\ springme@llnl.gov
}

\begin{abstract}
Designers, implementers, and marketers of data analysis tools typically have different perspectives than users. Consequently, data analysts often find themselves using tools focused on graphics and programming concepts rather than concepts which reflect their own domain and the context of their work. Some user studies focus on usability tests late in development; others observe work activity, but fail to show how to apply that knowledge in design. This paper describes a methodology for applying observations of data analysis work activity in prototype tool design. The approach can be used both in designing improved data analysis tools, and customizing visualization environments to specific applications. We present an example of usercentered design for a prototype tool to cull large data sets. We revisit the typical graphical approach of animating a large data set from the point of view of an analyst who is culling data. Field evaluations using the prototype tool not only revealed valuable usability information, but initiated in-depth discussions about users' work, tools, technology, and requirements.
\end{abstract}

\subsection{Introduction}

Visualization designers can enhance the usability of data analysis tools by tailoring the technology so tools reflect the scientist's world, rather than impose computer terminology or models. Yet, designers, implementers, and marketers of data analysis tools can not be expected to have expert knowledge of the work practices of every community for which they produce tools. Much tool development occurs to meet immediate needs and deadlines, without a concern for seeking or incorporating such knowledge. On the other hand, studies of work activity often end without following through to apply the newfound knowledge of practice in prototype tool design. The goal of this paper is to demonstrate how to bridge the gap between observations of work and tool design.

This paper builds on previous work which resulted in a methodology for observing and characterizing the scientific data analysis process [12]. Professional data analysts play a crucial role as domain experts throughout this design methodology, from observations to prototype evaluation. The software designer often assumes the role of an apprentice sitting in on an expert's work sessions while learning about the process to be supported. This paper reports how information gleaned using this designer-asapprentice approach was applied in prototyping a tool for an important element of data analysis: data culling.

We revisit the typical graphical approach of animating a large data set from the point of view of a data analyst who is culling data. For example, many tools organize data as a series of images and apply operations to one image at a time, whereas a data analyst may want to operate on the raw data over time, across variables, or on some arbitrary subset. We designed a tool to perform uscr-defined, formula-based queries on subsets of raw data, and contrasted it with the graphical analysis approach of a tool currently in use. Field evaluations with ten physicists and programmers using both tools not only revealed valuable usability information, but initiated in-depth discussions between the software designer and end users about users' work, tools, technology, and requirements.

There were two main goals for this study:

- to demonstrate the "designer-as-apprentice" prototyping methodology with its reliance on information from observations of work activity and field evaluations of a prototype tool;

- to examine an inadequately supported analysis task for which more effective support could have a substantial impact in terms of time saved and insight gained.

This paper is organized into sections on the concept of data culling, the prototype tool design, and the results of field exercises with physicists and developers.

As computers pervade the professionai world, many users have neither the time nor desire to adapt computer-centered tools to their work. Designers can apply the methodology described here to improve their analysis, design, and evaluation of prototypes to make the process more usercentered. Implementers, marketers, and support personnel could also benefit from a description of user work activity that shows how their products fit into the users' domain.

\subsection{Related work}

Several non-traditional design methodologies have emerged in recent years, each with a different approach to bringing the "user perspective" to bear on system design. Each approach shares the desire to distance itself from traditional systems design, with its focus on black box 
subdivision, and lack of concern for users and their environment. Cognitive theorists examine user interaction with tools, focusing on models which can predict user behavior [4]. In contrast, others have focused on social and environmental influences in designing for human activity, and have challenged the validity of the formal, analytical and predictive models of psychology $[14,2,20]$. Still other designers have noted the need for user participation in the design process itself [See 11] or the participation of designers in the users' environment [19].

The designer-as-apprentice approach focuses on the underlying process as practiced by users, rather than on refining one particular system or studying user behavior. The methodology shares characteristics of interaction analysis, with its focus on observation of actual work activity $[14,15,10]$. It also adapts the method of talking aloud [8], since data analysis is typically an individual activity. The designer-as-apprentice methodology calls for user participation throughout the full cycle of observation, understanding, and design.

Other related work includes the tools used in the evaluations [5] and analysis tools which have made possible graphical scanning of large amounts of data [e.g. 1, 7, 9, 18]. Also, managing large data sets has continued to be an important concern in scientific computing, and data culling is one aspect of this larger problem [16].

\subsection{Culling large data sets}

Culling data means finding and extracting areas of interest in large or complex data sets. As data sets grow in size, applying the techniques that are used on small data sets can become slow and awkward. In the environment for which the prototype tool was developed, a data set consists of a series of files, each representing one timestep in the evolution of a physical system. Table 1 describes four data sets used in the field evaluations. One of the sets contains 17 files; the other three contain one file each, and show how file size can vary by an order of magnitude or more.

A typical data set describes the interaction of several materials over time, defined on a rectangular mesh with 5 15 variables at each mesh point. Culling is the process of identifying the timesteps and regions of the mesh which

\begin{tabular}{|cccccr|}
\hline \multicolumn{6}{c}{ Sample Range of Data Set Size } \\
zones & vars & files & $\mathrm{mb}$ \\
1 & $373 \times 103=$ & $=38,419$ & 13 & 17 & 79.2 \\
2 & $251 \times 127=$ & $=31,877$ & 10 & 1 & 3.4 \\
3 & $479 \times 251$ & $=123,753$ & 10 & 1 & 12.8 \\
4 & $855 \times 275=235,125$ & 10 & 1 & 28.6 \\
\end{tabular}

Table 1. Range In size among typical flles. are of interest. For example, an analyst might decide to study the physical parameters of two particular materials after a collision has occurred. In some cases, finding this area might be the only goal. In most cases, analysis proceeds with the smaller portion of data once it is found.

\subsection{Culling approaches}

In initial analysis of a large-scale problem, knowledge from calculations or experiments may point to areas of interest. For example, a simulation code may report the timestep at which the collision in question occurred, so that one could isolate the data files surrounding it. If no such knowledge exists, one must jump from file to file, searching for the data of interest. This search typically occurs by viewing the contents with graphical analysis tools; the method could be to look at the entire set one-byone, to randomly select files, or to look at a file every so often in the series (e.g., every third file). Existing tools used in this process are not specifically designed for data culling, but can be applied in a piecemeal fashion.

In addition to culling data by decreasing the number of timesteps to be considered, several other reductions in size are possible. One could eliminate irrelevant zones in the grid, or variables and materials which are not of interest in the present analysis. The new, reduced data set would contain only data pertinent to the problem at hand. In particular, such a scheme for reducing large data sets would allow more use of workstations, which currently lack the resources (transfer rates, processing speed, memory) to adequately support large-scale scientific data analysis.

When trying to gain a qualitative understanding of the contents of a large data set, viewing an animation is often preferable to sorting through a large stack of numerical reports describing the same data. However, cases exist in which a scientist may have a quantitative or conditional understanding of the portion of data to be analyzed. For example, an analyst may want to know for which zones a certain value of pressure coincides with a certain density for some material. This type of event will not be easily identified in an animation, which is more suited to assisting in picking out a visual phenomenon such as the intertwining of two materials. While visualization is often crucial to data culling, some cases exist in which a nonimage-based approach might be even more effective. The prototyping effort focused on a number-based approach, in an attempt to identify such cases.

\subsection{Analysis tools of participants}

For the prototype design, we chose a class of data ior which analysis tools existed on a workstation and a supercomputer. The workstation tool MeshTV supports interactive analysis of rectangular or unstructured data [5]. Typical representations are pseudo-color and line contour plots of physical data, material boundary plots, mesh plots, and velocity-vector plots. The supercomputer program MTV is a postprocessor for data on a rectangular grid. 
MTV restricts graphics to black and white plots and provides a command line interface, while MeshTV takes advantage of workstation capabilities to offer a graphical user interface. Since it is logical to cull data on the machine that generates large data sets, the supercomputer program served as the starting point for the prototype tool.

Physicists use these analysis tools to generate plots of data for one variable represented on a grid at one point in time. Plots can be overlaid, so that a user could create a color contour plot of density and then overlay contour lines representing a related variable. For example, in Figure 1 color represents nuclei density and the contour lines overlaid on the plot and on an associated color bar represent the density of pions (dp), which are subatomic particles created in the nuclear collision process modeled with a hydrodynamics code. MTV plots of a variable, such as pressure, at different points in time can be generated and played in sequence to view an animation of how pressure evolves over time. Other plot series can show the shape of a material as it evolves, or boundaries of the grid.

MTV users can zero in on an area of interest in their data by generating and visually inspecting plots at individual points in time. Data sets are not reduced in terms of the size of the grid, or the number of variables or materials, although windowing functions exist to display subsets of a plot. MTV users described culling large data sets as a qualitative and fuzzy "grab-and-look" procedure, in contrast to the more quantitative and precise investigative

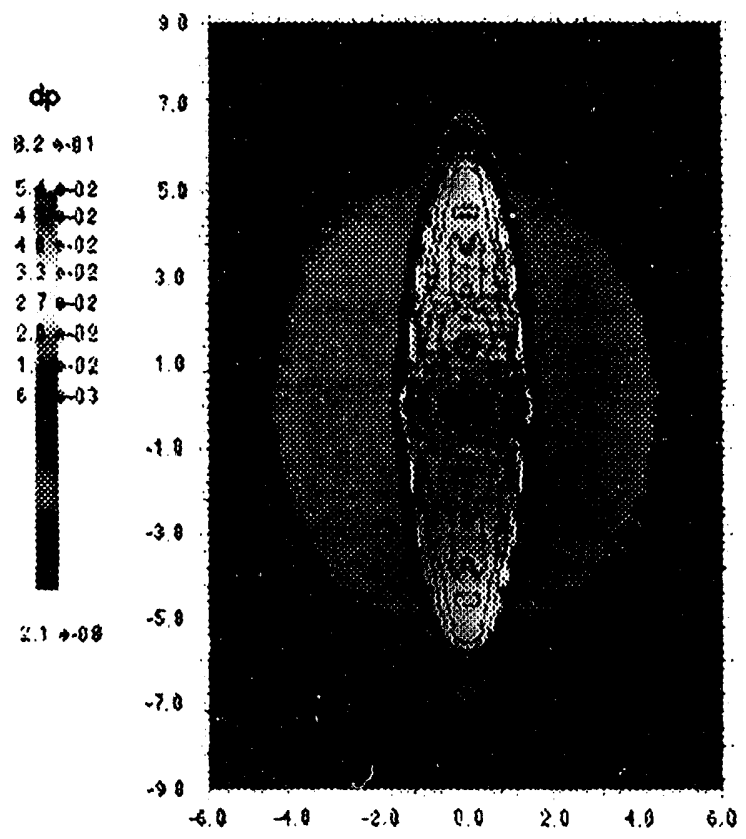

Figure 1. Mesh TV plots. Pion density contour lines are overlaid on a pseudocolor contour plot of nuclel density. techniques conducted by hand with smaller amounts of data. During observations in previous studies $[3,11]$, it became clear that more quantitative techniques might also benefit analysts in culling larger amounts of data. Furthermore, as design ideas began to take shape, scientists gave enthusiastic feedback on how this approach could improve the culling process.

\subsection{Observations of data culling}

Although there was not an abundance of data culling observed during the observation sessions of our previous study [11], several discussions about data culling took place. One physicist described how he currently culls data:

"That's a hard problem to deal with, and it's something we'd like to do a lot. For example, we'll do a calculation and watch a shape change in time....You'll want to see where shapes come from and follow them. The production codes will generate a bunch of data files. I can have a whole list of 1,000 of them or 10 of them and look at them in sequence [with a graphics post processor].... The other thing I can do is ask the code to make a sequence of graphics edits, and I can sit there and flip through them. Image is another way, if you can get [the data] into it. It's a case of none of the things really does what you want, completely. What's really the thing you'd want to do is not clear at all."

In further discussion, we asked this scientist whether being able to look for certain conditions in the data would be useful. He responded that such a program would be interesting, because sometimes people look through numerical records on microfiche to get such information.

\begin{abstract}
"When you look at the individual files, you're just grabbing a file -- well, let me try this one. Maybe it's around here.... What you'd like to be able to do is sort through 20 files and tell me when something happens. Or give me the value of temperature in this place in all these files. You can give me a plot of it and I can go and make a decision based on that. I mean, that might be an interesting tool to have."
\end{abstract}

In other sessions, scientists were observed to follow a similar sort of grab-and-look mode of searching for the right file, often frustrated to find that the chosen file was not the one sought. In cases where the problem resembles earlier runs, the culling problem becomes much easier.

\subsection{Comparing culling approaches}

An important purpose of the prototyping effort was to compare an image-based way of accomplishing data culling (visual search with MTV) with a number-based way (formulaic search with the prototype tool) in order to determine when one approach is more effective. It seemed clear from the observation sessions and interviews with 
scientists that a number-based approach could possibly be a more effective and faster solution to pinpointing a conditional area of interest. A main goal of the comparison was to learn the conditions under which this becomes true. Another goal of the effort was to explore with users how the number-based approach might be used to extend the culling and extraction capabilities of MTV and MeshTV.

The prototype tool, called MDC (Mesh Data Culler), adapted the MTV interface style so it would have similar commands for opening files, windowing, and asking for help. However, MDC was designed specifically for culling. Rather than sharing the MTV focus on plot specification (i.e. contours, material boundaries, vectors, and $x-y$ graphs), MDC focuses directly on culling data. The major commands involve searching and subselecting data. Results are reported in terms of culling tasks -- which zones met a condition, and for what time range?

The main function of the prototype tool is to perform searches for quantitative conditions on a series of files and within a grid subset. Users specify a formula, such as pressure $>$ value. The resulting report contains a set of labeled boundary curves, each curve showing the region that meets the condition for one file. Graphical analysis tools can be used to query for such conditions with a picture-bypicture search and user-adjusted contour levels. An imagebased tool is not geared to deal with a request like "show me where condition $X$ happens in every third file in a series." In contrast, MDC focuses directly on such tasks and summarizes the resuits over a series of timesteps.

\subsection{Prototype design issues}

Scientists participate in the prototyping effort throughout analysis, design, testing, and evaluations. The detailed characterization of scientific data analysis presented in [1] provided a firm foundation for considering observed data analysis activity and its bearing on the current design problem. Each category of the characterization was revisited from the perspective of how it might relate to data culling. This was not simply a one-pass, methodical checklist; the descriptions were consulted frequently throughout the design process. The knowledge gained in these earlier observations suggested functionality and usability issues to consider. For example, the issue of access to quantitative information, such as precise numerical values and coordinate locations, came up often in the observations.

In this section, we discuss four main issues addressed in the prototype tool design. The choices that we made were informed by our specific knowledge of user work activity. We also note graphics and interface theory which supports the decisions made in designing the prototype tool.

\subsection{Subselecting data on a mesh}

One way to focus on areas of interest within a single timestep concerns the grid on which the problem was defined. In many cases, much of the "action" occurs in only part of the grid, and the rest of the grid is carried along needlessly - from the point of view of post-processing. In image-based tools such data is often cropped out of images, but still exists in the original data file. One of the question considered was whether, given a knowledge of the relevant part of the grid, a scientist would want to specify that a search be restricted to that portion.

The specification of a grid subset is more work for a user, but speed may be a more important criteria. The MDC tool searches only that part of a grid which is specified, so selecting a small part of a grid can add up to significant savings when multiplied by the number of files. In cases of actually paring down data sets for shipment to MeshTV on a workstation, subselecting a grid has obvious benefits of reducing file size, data transport time, and data access time. The desirability of minimizing these costs is clear [16]. Another consideration is that to view the whole grid can often mean to view much information unnecessary to the task at hand. Tufte [17] discusses the advantages of presenting only that portion of data relevant to the task.

\subsection{Providing time-dependent operations}

Much scientific data, including that used in this study, represents an evolution over time. Data sets are typically structured as a series of files, one file per timestep. Analysis tools typically operate on one file at a time and the user repeats a set of actions for each file. For example, to find the minimum or maximum value of a variable, one would manually ask for the values for each file. From the point of view of culling the data set, one would like to apply such an operation to an entire class or subset of files.

The MTV program has a command, "plotnext," for repeating a plotting operation for the next file in a series of files sharing the same prefix. This alleviates having to open the file and respecify the plot, thereby offering a useful shortcut. However, it also demonstrates the repetitive effort required of users to manually call up each file albeit by only typing a letter or two (i.e. " $p$ " to plot the next file, or "p+2" to plot the file after next). By applying a command to an entire class of files, designers can lower repetition and increase efficiency. The criterion of efficiency applies to speed and compactness of queries as well as the increased speed made possible through reduced interaction. That is, the MDC tool can open, query, and close a series of files in rapid succession. This speed decreases greatly when each file is specified one by one and the program must stop to wait for user input in between operations.

If one chooses to provide set operations, this opens a new question of how to specify a filelist. A simple approach would be to select all files with a certain prefix; this would be easy, but not flexible. In contrast, a second option would be to build a list. Any combination of files sharing the same grid dimensions and variables could be included in a family of files. However, the specification of such a list would be somewhat complex. A compromise between these two approaches would be to select a subset according to a stride between files. That is, the filelist consists of every $n$th file with a matching prefix. (For example, 
building a filelist with a stride of 2 from the files mic 00000 , mic 00001 , mic00002, mic00003 would include the files ending in even numbers). This latter option is more flexible than including all files in the set and is easier to use but somewhat less flexible than laboriously specifying the filelist one file at a time.

\subsection{Presenting evolution over time}

Three possible options were considered for how to present the results of a culling request. The first, a usercontrolled one-by-onis march, allows great variety in the examination order ir. which individual timestep results are viewed. Other iriteria include an ability to convey complex visual motior, and an ability to convey an evolution in one image. Although a one-by-one march excels in variety of order, it fails to meet either of the other criteria.

A second option, animation, would be the most popular suggestion to solve data culling problems. Particularly in cases of complex visual events, such as weather modeling (e.g. the development of a thunderstorm), animations clearly provide an excellent means of understanding an evolution over time. However, looking at one image or frame of an animation provides little insight into the evolution. The third option, a composite of timesteps on one image, addresses cases in which a simple evolution over time can be depicted. In the case of data culling, we predicted that such cases would lend themselves to a composite image.

\section{Mesh Data Culling (MDC) Command Summary}

\section{Search Commands}

find var op value I varl op 1 valuel logop1 var2 op2 val2 logop2 ...

Op: $<=,>=,<,>, \quad=$, closeto

Logop: and, or

minmax $\operatorname{var} 1 \operatorname{var} 2 \ldots \operatorname{var} \mathrm{N}$

Set-up Commands

open filename

filelist beginfile endfile stride | all | beginfile stride grid $k 1$ k2 j1 j2

$\log$

longlog

plot curves l marker Ifill

Plot Commands

window $x 1 \times 2$ y 1 y2

windowkj $k 1 \mathrm{k} 2 \mathrm{j} 1 \mathrm{j} 2$

resetwin

Information Commands

help topic1 topic2 .. topic n

list dump filelist vars commands grid plot

end

Figure 2. Functions of the data culling tool.

\subsection{Providing quantitative information}

Providing adequate access to quantitative information was one of the major design recommendations in [13]. We considered the trade-offs of a brief report on the screen versus a detailed history file. The file would contain more quantitative detail, and could be viewed and discarded or kept as part of analysis records. The level of detail automatically generated for the screen display was an issue closely examined during testing and the exploratory exercises. The report was changed several times in response to user feedback. The trade-offs can be noted as two desirable but conflicting criteria: minimal storage versus fine level of detail. A compromise was to include both types of reports, and to allow two levels of detail in the history file.

\subsection{Prototype tool functionality}

The main function of the prototype tool is to perform quantitative searches for a condition on a series of files and within a grid subset. The resulting display contains a set of labeled boundary curves, showing the region that meets the condition for each file. A user can specify, for example, that the tool should find the minimum and maximum values for temperature, pressure, and density in a series of files. Or find the areas where one variable is precisely equal to 0 and another exceeds 5.68. Secondary functions include turning on a report mechanism, listing information and help, changing the plot style or window size, and setting other minor parameters.

The MDC tool was designed to be consistent with the MTV user interface, to ensure a valid comparison. Certain commands share the same syntax, such as those to open files, set windows, and ask for help. The command list in Figure 2 summarizes the functionality of the MDC tool.

\section{Simple Culling Task with MDC}

Example. Find where pressure exceeds 0.04 for every eighth file in the "mic" series of files.

mdc

$\rightarrow$ filelist mic00000 8

Current dump $=$ mic00000; $373 \times 103,38419$ zones;

Time $=1.20056 \mathrm{e}+01$

Selected 3 files: mic00000 to mic01104, stride 8

$\rightarrow$ list vars

13 variables available:

\begin{tabular}{|c|c|c|c|c|c|}
\hline $\begin{array}{l}\text { u } \\
\text { gy }\end{array}$ & $\begin{array}{l}v \\
\text { urr }\end{array}$ & $\begin{array}{l}d \\
\text { tzz }\end{array}$ & $\begin{array}{l}\text { te } \\
\text { trz }\end{array}$ & $\begin{array}{l}\text { ist } \\
\text { eps }\end{array}$ & $p$ \\
\hline find & $\begin{array}{l}p \\
\text { filename }\end{array}$ & & numb & of zones & \\
\hline & $\begin{array}{l}\operatorname{mic} 00000 \\
\text { mic00449 } \\
\text { mic01104 }\end{array}$ & & & $\begin{array}{r}1562 \\
904 \\
0\end{array}$ & \\
\hline
\end{tabular}

Figure 3. Example data culling operation. 
An example of a simple culling task appears in Figure 3. The user instructs MDC to select every 8 th file in the series of files beginning with the letters "mic." MDC responds by opening the filelist and reporting on the mesh size, the time of the first file in the list, and the number of files in the list. When queried for the variables available in that series of files, MDC responds with the number of variables and a list. Given that information, the user instructs MDC to find all zones for which pressure exceeds the value 0.04 . As MDC prints the number of zones found for each file in the filelist, a curve marking the boundary of those zones is displayed. The final image, shown in Figure 4 , contains a view with only two boundaries, since one of the files had no zones for which pressure $>0.04$ was true.

In addition to plots, users may print out or display "logs," or reports about the particular zones which meet the condition. Zones can be designated by logical position within the mesh structure $(k, j)$, or by absolute coordinates in space $(x, y)$. A short report contains the $(k, j)$ and $[x, y]$ positions of two zones. (These are the first and last zones that met the condition; although dependent on the array ordering, this provides some quantitative feedback). One entry appears in the table for each file (i.e., timestep). A longer report can be generated (iusing longlog) which contains the entire list of positions for each zone meeting the condition, and the value of the first variable in the formula. These reports can be used as intermediate, on-line results or can be kept as records of the data analysis session.

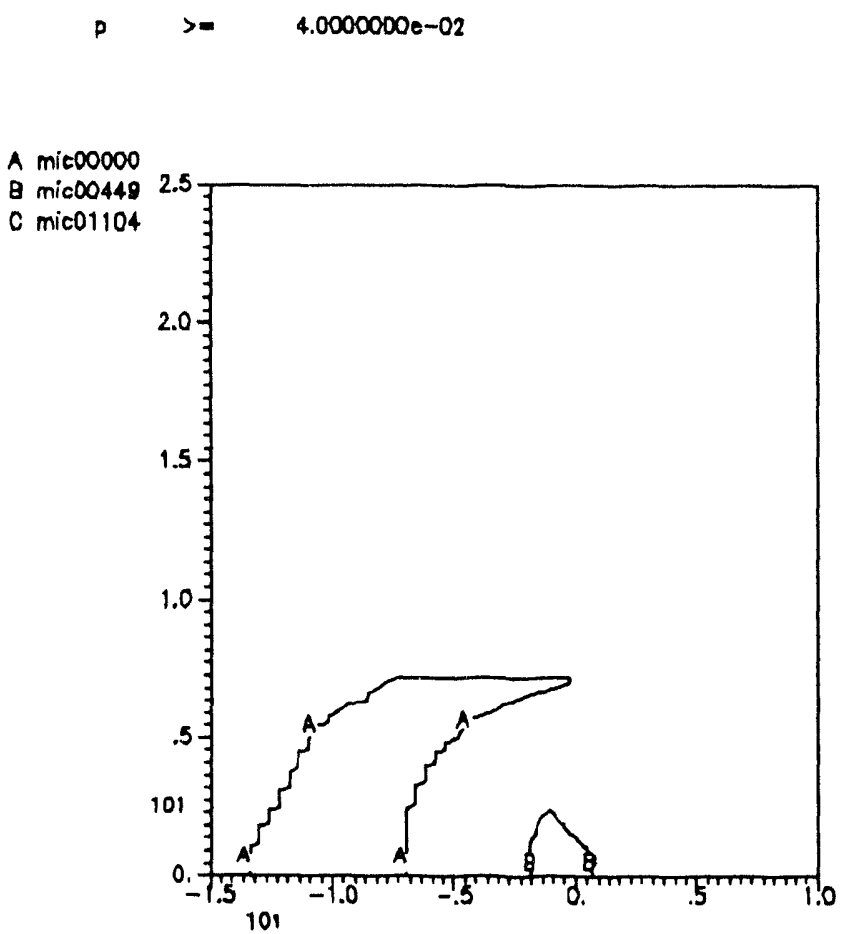

Figure 4. The plot resulting from the find command in the example of Figure 3.

\subsection{Development and testing}

The purpose of the prototype was to probe, compare, and evaluate, not to redesign and develop an entirely new system. Heavy software re-use and an interface familiar to the users allowed experimentation using nearly off-the-shelf tools to speed development. Software re-use included several library routines for parsing commands and reading the large database "dumps" produced by the simulation code at pre-specified timesteps. Portions of the MTV code served as a model for handling the filelists and graphics devices. The MDC program was debugged and tested with real data sets to ensure the correctness of the program.

Feedback from initial testers (a computer scientist and a physicist) was used to improve the functionality and user interface of the MDC prototype tool. Changes ranged from simple alterations of command names to modifications in functionality. For example, boundary plots were unable to display the precise shape formed by zones meeting an MDC condition. A filled plot was developed to serve this purpose, and was promptly used by the physicist on one of his problems. Thus, development and testing led to both a refined tool as well as a better understanding of possible data culling tasks. This understanding was extended with the evaluation exercises described in the next section.

\subsection{Field evaluations}

A series of ten prototype evaluation sessions was conducted with eight MTV users. Participants included two physicists who develop simulation codes, four physicists who use those simulation codes in their work, and two programmers. Each of the six physicists routinely analyzes large data sets with MTV as well as other tools. The two programmers use MTV in their role as developers and maintainers of scientific software tools.

Each session began with an introduction to MDC. Part cipants were given a four-page explanation of the comı،ands and a one-page syntax summary. An on-line 15 20 minute practice session provided participants with handson experience and an opportunity to ask questions while using the tool. Participants were then asked to talk aloud [8] while completing an exercise which took about 30 minutes. The exercise included culling tasks such as searching for areas with variable values higher than some threshold, or finding the minimum and maximum values for a set of variables. Some participants also used the tool to perform tasks on their own data sets. Throughout the session, the observer watched, took notes, and answered any questions. The last five sessions were audiotaped to provide a more detailed record. The tapes were then transcribed and analyzed along with the notes from all the sessions.

\subsection{User feedback}

Selected comments made by participants during the prototype evaluations appear in Figure 5. More detailed comments and a description of the method of evaluation 
appear in Chapter 6 of [13]. Here, we summarize main points made by participants about data culling, the MDC approach, and the users' environment.

Most of the comments about the MDC tool and approach were positive, although several people pointed out possible improvements. In particular, the users would like to see MDC include all the MTV plot types. (These were not implemented as part of the prototype, since the goal was not a complete data culling tool, but a prototype to test out the number-based approach).

Several comments indicated that the ability to operate on whole sets of data was helpful and would help save time. In addition, the MDC presentations on both the screen and in $\log$ reports provided analysts with tabular summaries of complete data sets unavailable in other tools.

Most participants were enthusiastic about the numberbased functionality. A numerical summary of the search results provides a way to get a quantitative view of several variables at once. In this particular environment, it was the only tool which could provide this important quantitative overview. (Users currently handle such tasks manually.)

Participants said that the number-based approach was particularly important for checking out errors in calculations or while debugging. It works well for isolating points which are too close to tell apart in an image, since the $\log$ function of MDC provides the floating point numbers from the raw data.

Several comments indicated that we had provided an interface which more closely modeled the user's work activity than other interfaces. For example, during the sessions users were focused on culling tasks while using the MDC tool. In contrast, while using MTV some users spent time focused on plotting, windowing, and opening files rather than on data culling tasks.

Comments about extending these ideas are included in Figure 5, to demonstrate the variety of information offered

\section{On queries over time to reduce repetition:}

The filelist capability is definitely useful, because right now it's really a pain to try and step through the files. You really just want to treat all of them as one block.

This would be very useful for finding where things were going haywire in a whole bunch ... without opening each file separately.

The things that I've seen are useful [because] I don't have to be so repetitious.

Extremely useful.

What strikes me as most useful is the ability to watch a propagation over time.

\section{On supporting number-based tasks:}

This would be useful for damaged files, to find where there is a peculiar boundary between two materials.

You might want to find out where's the extreme, if things go bad. We might well look at every zone in a small problem and kind of get a detailed picture.

I would like to specify what variables to pick up. Tell me the physical coordinates, pressure, temperature, energy, and density [at each point meeting the criteria].

Can you actually manipulate what you found? [i.e. Can you perform arithmetic on items in the log?].

I wouldn't want a longlog with 2000 points. Might want to be able to turn on a "verbose mode" to show some of the short log information on the screen.

I really like being able to use arithmetic expressions to scan through things. Right now in MTV that's pretty hard, because it's built on top of the graphics. And here we're really trying to do mathematical operations.

\section{On whether logical formulas are useful:}

Yes, yes absolutely. You might like to ask yourself combinations of questions. Did this and this occur, and that's why I got to this state?

Yeah, that's exactly what I want. In fact, that would be perfect. Let's do find $t>=0.05$ and $d>=.10$. What you are seeing now are contours that don't go so far out. So this is very handy. It shows you lots.

Oh yeah, I'd probably find uses for it. It would be extremely useful in some cases.

It doesn't occur to me right now; I would have to get used to it. Let's look at [in the user's data ] $u>1.0$.

It's particularly useful for debugging things.... I would also like to have all the variables at a particular zone. That will give me some idea of what's going on.

\section{On extending these Ideas in future work:}

I think that it would be useful on the Cray. I wouldn't want to stop there.

You could certainly see the advantages of [moving MDC to the workstation], too.

You might be able to feed information back to the code .- to say, wait a minute, if this has happened, stop. And you could save hours of [wasted] computing time.

What I would like to do is say, for this material, find all the copper in the problem that's moving faster than .3 centimeters a microsecond. From the physics, I know it's not all here in left field. So I can look at the problem and say there's nothing anomalous going on.

What I' $m$ doing [by hand] now is one plot versus another. This is velocity vs. position, and this is integrated mass vs. position. Now I want mass versus velocity, over time.

\section{Figure 5. Evaluation session comments about the data culling prototype tool and approach.}


by participants. Such feedback follows naturally from working with users to evaluate exploratory prototype tools. whereas a user would not likely report such thoughts in response to a request to state needs and requirements. The evaluation sessions were successful as a springboard from which to begin a guided tour of data culling activity. Half of the physicists decided to try out the prototype tool on their own data during the sessions. The others talked about how they would use it. The guided tours of culling tasks led to interesting and valuable discussions of how MDC was useful to the analyst in ways that MTV was not.

\subsection{Conclusion}

This study provides a rich source of information for people who design systems to support data analysis as well as data culling. More importantly for designers in general, it demonstrates how a designer's understanding of a complicated, creative process can be enriched by working closely with professionals as they practice that process. In order to keep users interested in participating in the design and evaluation of prototype systems, sessions should be conducted in the context of the user environment.

The design approach of this work shifts the focus of prototype evaluation away from evaluating intricacies of a user interface or system performance, and onto the underlying process which is to be supported. Designers can not be expected to be familiar with the work practices of every user community for which they may design. Thus, the designer-as-apprentice methodology for gaining a process understanding which evolves throughout observation, characterization, and evaluation should be a useful tool for many designers.

\section{Acknowledgments}

The image in Figure 1 was contributed by Tom McAbee of Lawrence Livermore National Laboratory. This work was performed under the auspices of the U.S. Department of Energy by LLNL under contract No. W-7405-Eng-48.

\section{References}

1. Bancroft, G., F. Merritt, T. Plessel, P. Kelaita, R. McCabe, A. Globus, "FAST: A Multi-Processed Environment for Visualization of Computational Fluid Dynamics," in Proceedings of IEEE Visualization '90, San Francisco, California, pp. 14-24, October 1990.

2. Bodker, S., Through the Interface: A Human Activity Approcich to User Interface Design, Hillsdale, NJ: Lawrence Earlbaum Associates, 1991.

3. Cabral, 3. and Hunter, C., Visualization Tools at Lawrence Livermore National Laboratory, IEEE CG\&A, Vol. 22, No. 8. Augus: 1989, pp. 77-84.

4. Card, S.K. T. P. Moran, and A. Newell, The Psychology of
Human Computer Inseraction, Lawrence Earlbaum Associates. Hillsdale, New Jersey, 1983.

5. Cook, L. and J. Long, "An Interactive Visualization System for Scientific Data," LLNL Energy and Technology Review, UCRL-52000-90-1, January/February, 1990.

6. Dyer, D. S. A Dataflow Toolkit for Visualization, IEEE CG\&A, Vol. 10, No. 4, July $19,0$.

7. Ericsson, K. A. and H. A. Simon, "Verbal Reports as Data", Psychological Review, 87, 1980, pp. 215-251.

8. "IRIS Explorer," Silicon Graphics Computer Systems Technical Report, 1991.

9. Minneman, S. L., The Social Construction of A Technical Reality: Empirical Studies of Group Engineering Design Practice, Xerox PARC Technical Report SSL-91-22 (Doctoral Dissertation, Stanford University), December 1991

10. Namioka, A., and D. Schuler (eds.), 1990 Participatory Design Conference Proceedings, Seattle, WA, April, 1990.

11. Springmeyer, R., M. Blaltner, N. Max, "A Characteriza-tion of the Scientific Data Analysis Process," Proceedings of IEEE Visualization '92, October 1992, pp. 235-242.

12. Springmeyer, R. Designing for Scientific Data Analysis: From Practice to Prototype. Ph.D. Thesis, University of California, Davis, September 1992.

13. Suchman, L. Plans and Situated Actions: The problem of human-machine communication, Cambridge: Cambridge University Press, 1987.

14. Tang, J. and Leifer, L. Observations from an Empirical Study of the Workspace Activity of Design Teams, in Proceedings of the First International ASME Conference on Design Theory and Methodology, September, 1989.

15. Treinish, L., "SIGGRAPH '90 Workshop Report: Data Structures and Access Software for Scientific Visualization," Computer Graphics, Vol. 25, No. 2, pp. 104-118, April 1991.

16. Tufte, E. R., Envisioning Information, Cheshire, CT: Graphics Press, 1990.

17. Upson, C., T. Faulhaber, D. Kamins, D. Laidlaw, D. Schlegel, J. Vroom, R. Gurwitz, A. van Dam, The Application Visualization System: A Computational Environment for Scientific Visualization, IEEE Computer. July 1989.

18. Whiteside, J., J. Bennett, and K. Holtzblatt, "Usability Engineering: Our Experience and Evolution, Handbook of Human-Computer Interaction, M. Helander (ed.), NorthHolland: Elsevier Science Publishers, pp. 791-817, 1988.

19. Wixon, D. and K. Holtzblatt, "Contextual Design: An Emergent View of System Design," SIGCHI '90 Conference Proceedings, pp. 329-336, 1990. 
11
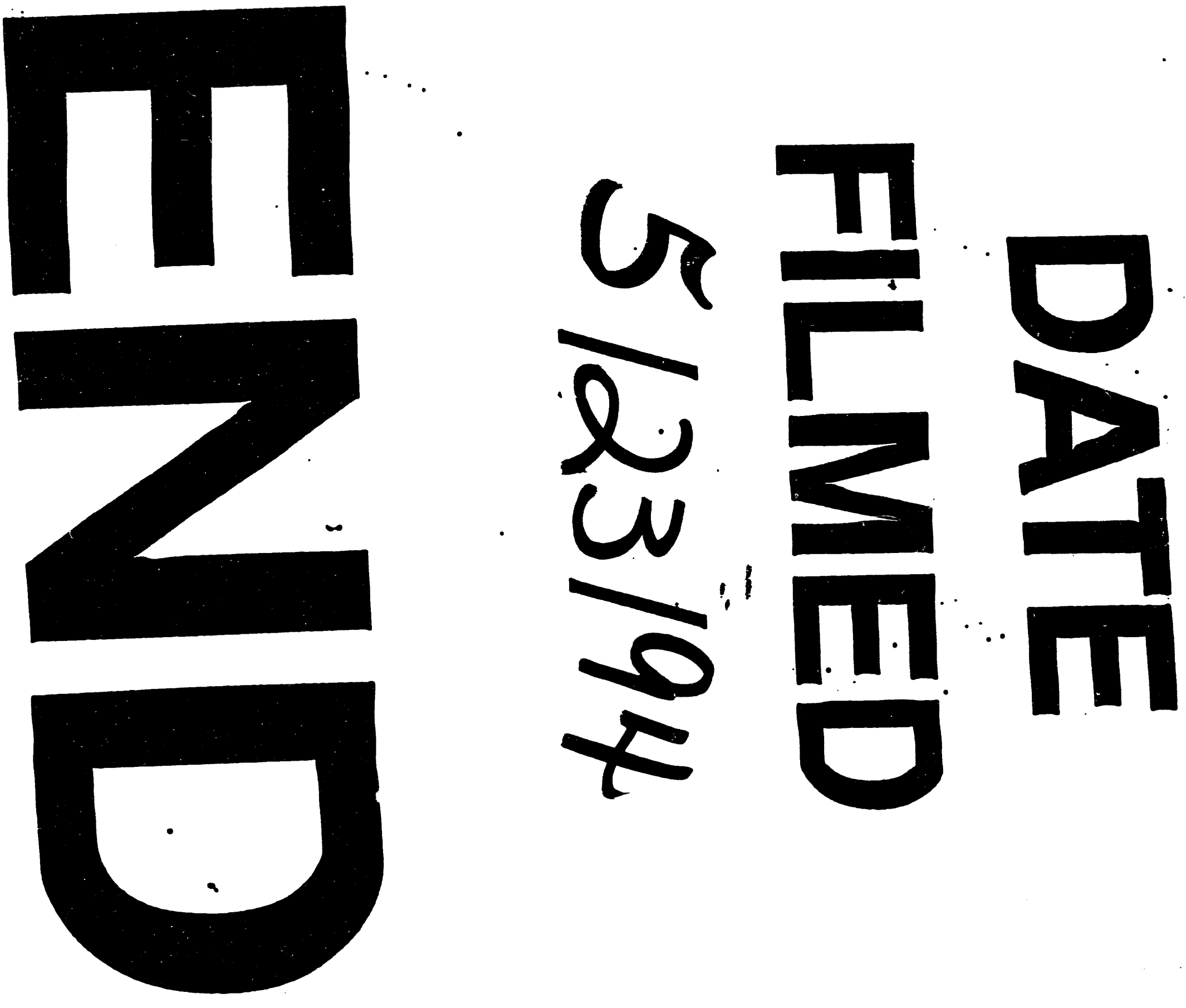